\title{
Acknowledgment to reviewers who served in 2011
}

(c) The Japanese Society for Hygiene 2011

We highly appreciate the kind contribution of the following individuals who served as reviewers for articles submitted to Environmental Health and Preventive Medicine in 2011.

\author{
Akiba, Suminori (Kagoshima) \\ Asakura, Keiko (Tokyo) \\ Babazono, Akira (Fukuoka) \\ Fujiwara, Takeo (Tokyo) \\ Fukuda, Yoshiharu (Yamaguchi) \\ Fukunaga, Ichiro (Sakaide) \\ Hamazaki, Kei (Toyama) \\ Hata, Akira (Chiba) \\ Hirano, Seishiro (Tsukuba) \\ Hirata, Mamoru (Amagasaki) \\ Honda, Yasushi (Tsukuba) \\ Hori, Reiko (Aichi-gun) \\ Horiguchi, Hyogo (Akita) \\ Horiuchi, Masahisa (Kagoshima) \\ Ichihara, Gaku (Nagoya) \\ Ichihara, Sahoko (Tsu) \\ Inoue, Yusuke (Tokyo) \\ Ishikawa, Hirofumi (Okayama) \\ Ishikawa, Sho (Tokyo) \\ Ishizaki, Tatsuro (Tokyo) \\ Izawa, Tetsuya (Kyotanabe) \\ Kamada, Masamitsu (Tomi) \\ Kamijima, Michihiro (Nagoya) \\ Kanamori, Masao (Otsu) \\ Kishi, Reiko (Sapporo)
}

Kobayashi, Nobumichi (Sapporo)

Kojima, Masayo (Nagoya)

Koyama, Hiroshi (Maebashi)

Kuroda, Tatsuhiko (Tokyo)

Matsubayashi, Kozo (Kyoto)

Michikawa, Takehiro (Tokyo)

Miyai, Nobuyuki (Kashiwara)

Miyashita, Kazuhisa (Wakayama)

Morita, Emi (Nagoya)

Nagaya, Teruo (Nagoya)

Nakai, Kunihiko (Sendai)

Nakaji, Shigeyuki (Hirosaki)

Nakamura, Hiroyuki (Kanazawa)

Nakamura, Keiko (Tokyo)

Nakamura, Koshi (Kanazawa)

Nakamura, Yosikazu (Shimotsuke)

Nakano, Norihiko (Ibaraki)

Nakayama, Kunio (Suita)

Nasu, Tamie (Nagoya)

Ogawa, Masanori (Shimotsuke)

Ohkado, Akihiro (Kiyose)

Okubo, Yasushi (Tokyo)

Okuda, Masayuki (Yamaguchi)

Ono, Rei (Kobe)

Oono, Hideki (Mitaka)
Akio Koizumi

Editor-in-Chief

Saito, Takeshi (Sapporo)

Sakamoto, Tatsuo (Kawasaki)

Shima, Masayuki (Nishinomiya)

Shinohara, Atsuko (Tokyo)

Sowa, Yoshihiro (Kyoto)

Suganuma, Narufumi (Nankoku)

Sugiura, Minoru (Tsukuba)

Sunagawa, Tomimasa (Tokyo)

Suwazono, Yasushi (Chiba)

Takatorige, Toshio (Suita)

Takeshita, Tatsuya (Wakayama)

Tanaka, Keitaro (Saga)

Tanaka, Masatoshi (Fukushima)

Toda, Masahiro (Osaka)

Tsuda, Toshihide (Okayama)

Ueda, Kayo (Tsukuba)

Wakabayashi, Ichiro (Nishinomiya)

Yamagata, Zentaro (Chuo)

Yamauchi, Hiroshi (Tokyo)

Yatsuya, Hiroshi (Nagoya)

Yokoyama, Kazuhito (Tokyo)

Yonemoto, Junzou (Tsukuba)

Yoshida, Takahiko (Asahikawa)

Yoshimasu, Kouichi (Wakayama)

Yoshioka, Eiji (Sapporo) 